\title{
Rapid Analysis of Network Connectivity
}

\author{
Scott Freitas \\ Arizona State University \\ Tempe, Arizona \\ scott.freitas@asu.edu \\ Nan Cao \\ Tongji University \\ Shanghai, China \\ nan.cao@nyu.edu
}

\author{
Hanghang Tong \\ Arizona State University \\ Tempe, Arizona \\ hanghang.tong@asu.edu \\ Yinglong Xia \\ Huawei \\ Santa Clara, California \\ yinglong.xia@huawei.com
}

\begin{abstract}
This research focuses on accelerating the computational time of two base network algorithms (k-simple shortest paths and minimum spanning tree for a subset of nodes)-cornerstones behind a variety of network connectivity mining tasks-with the goal of rapidly finding network pathways and trees using a set of user-specific query nodes. To facilitate this process we utilize: (1) multi-threaded algorithm variations, (2) network re-use for subsequent queries and (3) a novel algorithm, Key Neighboring Vertices (KNV), to reduce the network search space. The proposed KNV algorithm serves a dual purpose: (a) to reduce the computation time for algorithmic analysis and (b) to identify key vertices in the network (context). Empirical results indicate this combination of techniques significantly improves the baseline performance of both algorithms. We have also developed a web platform utilizing the proposed network algorithms to enable researchers and practitioners to both visualize and interact with their datasets (PathFinder: http://www.path-finder.io).
\end{abstract}

\section{KEYWORDS}

k-simple shortest paths, MST, search space reduction, multi-threading, parallel processing, network visualization, seed nodes

\section{INTRODUCTION}

Motivation. With the advent of the big data era and the emergence of network science, large-scale networks are appearing across many disciplines, from medicine and epidemiology to advertising and marketing. As a result, an exponential amount of network data is being generated at an unprecedented rate. The challenge before us, given limited computational resources, is to translate this large network data into meaningful knowledge.

Problem. How can we rapidly explore, analyze and visualize a set of user-specific query nodes in relation to a dataset? We envision that tree, pathway and context are the three key components to answer this question. Formally, given a graph $G=(V, E)$ and a set

Permission to make digital or hard copies of all or part of this work for personal or classroom use is granted without fee provided that copies are not made or distributed for profit or commercial advantage and that copies bear this notice and the full citation on the first page. Copyrights for components of this work owned by others than the author(s) must be honored. Abstracting with credit is permitted. To copy otherwise, or republish, to post on servers or to redistribute to lists, requires prior specific permission and/or a fee. Request permissions from permissions@acm.org.

CIKM'17, November 6-10, 2017, Singapore, Singapore

(ㄷ) 2017 Copyright held by the owner/author(s). Publication rights licensed to Association for Computing Machinery.

ACM ISBN 978-1-4503-4918-5/17/11 . \$ \$15.00

https://doi.org/10.1145/3132847.3133170 of user-specific query nodes $Q$, we seek to find (1) the relationship between each query node in $Q$ (i.e. tree detection), (2) a subset of paths $R \subset G$ such that $R$ contains only vertices and edges that provide key path information between different query nodes in $Q$ (i.e. pathway detection) and (3) a subset of important vertices, $C$ and edges, $S$, such that $C \subset V$ and $S \subset E$ (i.e. context detection).

Related Work. There has been significant research related to our proposed algorithms. In addition, the concept of user-specific query nodes (seed nodes) has been an active research topic. For example, Staudt et. al. used user-specific query nodes for community detection via "selSCAN" [2] and Akoglu et. al. used them to find connection pathways [1].

K-Simple Shortest Paths (pathway): A theoretical analysis has been laid out by Ruppert [4], along with the theoretical and experimental work by Guerriero et. al using a shared memory model [5]. More recently, Singh et. al. used GPUs along with CUDA to parallelize the algorithm, resulting in impressive speedup [6]. In addition, two representative works for connection subgraph identification (i.e. pathway detection) can be seen in [1] and [3].

Shortest Paths MST (tree): Related work towards creating a minimum spanning tree for a susbset of nodes has been done by Cenek et. al. Instead of creating a shortest paths distance matrix, they proposed to insert the shortest paths into the tree dynamically [8].

Key Neighboring Vertices (context): Sulieman et. al. proposed a semantic social breadth first search algorithm which takes the top $N$ vertices with the highest centrality degree and attempts to find the nearby influential players [7].

Contributions. Our main contributions are three-fold: (1) the development of two multi-threaded algorithms: k-simple shortest paths (KSSP) and minimum spanning tree for a subset of nodes (Shortest Paths MST); (2) the creation of a novel algorithm, Key Neighboring Vertices (KNV), for reducing network search space and identifying key vertices; (3) the development of a web platform, utilizing these algorithms, for researchers and practitioners to upload their own network data for analysis and visualization.

1. K-Simple Shortest Paths (pathway): Our approach to the ksimple shortest path algorithm offers three new features: (a) instead of creating a multi-threaded single source shortest path algorithm [4][5][6], we parallelize each single source shortest path computation required to find a path in $k$ (for details see section 3.1); (b) we pre-process the network data to reduce the search space using the KNV algorithm; (c) the generated pathways and surrounding context nodes can be re-queried to find additional paths or trees. 
Shortest Paths MST (tree): Our approach to solving the minimum spanning tree for a subset of nodes (Shortest Paths MST) is centered around (a) parallelizing the shortest path computations to run simultaneously; (b) pre-processing the network data to reduce the search space of the algorithm; (c) re-querying the generated tree and surrounding context nodes to find additional trees or paths.

2. Key Neighboring Vertices (context): Our approach is similar to Sulieman et. al., but with three key differences. (a) Instead of exploring the network based on the top $N$ vertices with highest degree centrality, we seed the map with a set of user-specific query nodes; (b) we propose using above average network degree centrality as the metric for including nearby neighboring vertices for further exploration in the search list; (c) we allow additional parameters that let the user control the exploration process.

3. Platform: We have developed PAThFinder, a web platform to assist users in mining network connectivity from large networks. PATHFInder begins by taking an input network uploaded by the user or selection from a pre-loaded dataset. Depending on the user's expertise with the program there are two sets of controls: basic and advanced. The basic controls allow the user to start without an understanding of the algorithms, while the advanced controls allow the user to fine-tune their queries and obtain information that may not be available with a basic search. Visualizations are generated using vis.js and GraphViz. A video demo of the platform is available at: https://youtu.be/PxQVd-6mKUw.

\section{PLATFORM FUNCTIONALITY}

Each part of Figure 1 highlights some of the platforms core functionality. Figure 1(1) shows a sample visualization using the pathway detection algorithm on the DBLP network. Figure 1(2) allows the user to enter the algorithm parameters and select a network for analysis. Figure 1(3) allows the user to enter nodes and edges to be removed from the graph search, select whether or not to re-use the current graph results for further analysis and change the configuration of network style parameters, including: node-edge color scheme and node size. Figure 1(4) is a zoomed in portion of Figure 1(1). The red nodes represent the start and end query vertices, orange for intermediate path vertices, blue for one hop away critical vertices and purple for two hop away critical vertices. The blue and purple vertices surrounding the the path vertices are determined by the KNV algorithm.

Engaging the Audience. We expect that our demo will primarily attract two audiences, (1) practitioners who are interested in exploring the connectivity between key nodes in large networks, and (2) information management and data mining researchers who develop new algorithms and tools.

\section{TECHNICAL DETAILS}

All algorithms perform on an undirected, unweighted, adjacency list graph representation, $G=(V, E)$. Nonetheless, we note that the proposed platform is flexible to admit alternative algorithms and graph types with mild changes.

\subsection{Pathway Detection}

Problem definition. Given two pre-marked vertices, $x, y \in V$ from the graph $G=(V, E)$, this algorithm will find k-simple shortest paths from $x$ to $y$.
Algorithm description. We adopt the $k$-simple shortest paths threaded and search reduced algorithm (Algorithm 1) to detect key pathways that connect the query nodes, which can be viewed in 7 steps:

(1) Run the Key Neighboring Vertices (KNV) algorithm to reduce the search space of the graph using the start and end vertices.

(2) Find the first single source shortest path between vertex $x$ and vertex $y$.

(3) Run the KNV algorithm a second time on the original graph with all the vertices from the shortest path.

(4) For each edge in the current shortest path: temporarily remove the given edge and run a single source shortest path algorithm. Each of the shortest paths run in parallel.

(5) Determine which of these paths produced the subsequent shortest path. Permanently delete the edge that formed the shortest path from the adjacency list.

(6) Repeat Steps 4-5 until $k$ shortest paths have been found or there are no more identifiable paths.

(7) Optional: Re-query the generated network for additional paths, paths between different vertices or for a tree using Shortest Paths MST.

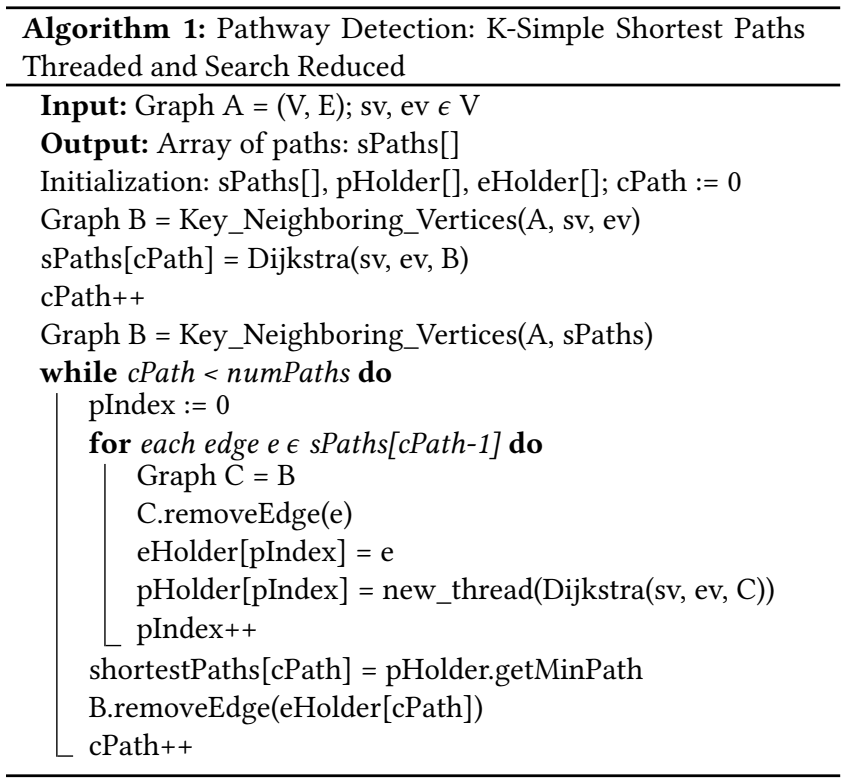

\subsection{Tree Detection}

Problem definition. Given two or more pre-marked vertices, $2 \ldots x$ $\epsilon V$ from the graph $G=(V, E)$, this algorithm will find a MST that is constructed from a combination of single source shortest paths. Algorithm description. We adopt the Shortest Paths Minimum Spanning Tree algorithm (Algorithm 2) to determine the relationship between the user-specified query nodes. This can be viewed in 4 steps:

(1) Each pre-marked node, $v$, will run a single source shortest path algorithm against every other pre-marked vertex. The single source shortest path algorithms run in parallel.

(2) Sort the resulting paths in ascending order of path length.

(3) Run Kruskal's algorithm to determine which of these shortest paths form the MST. 


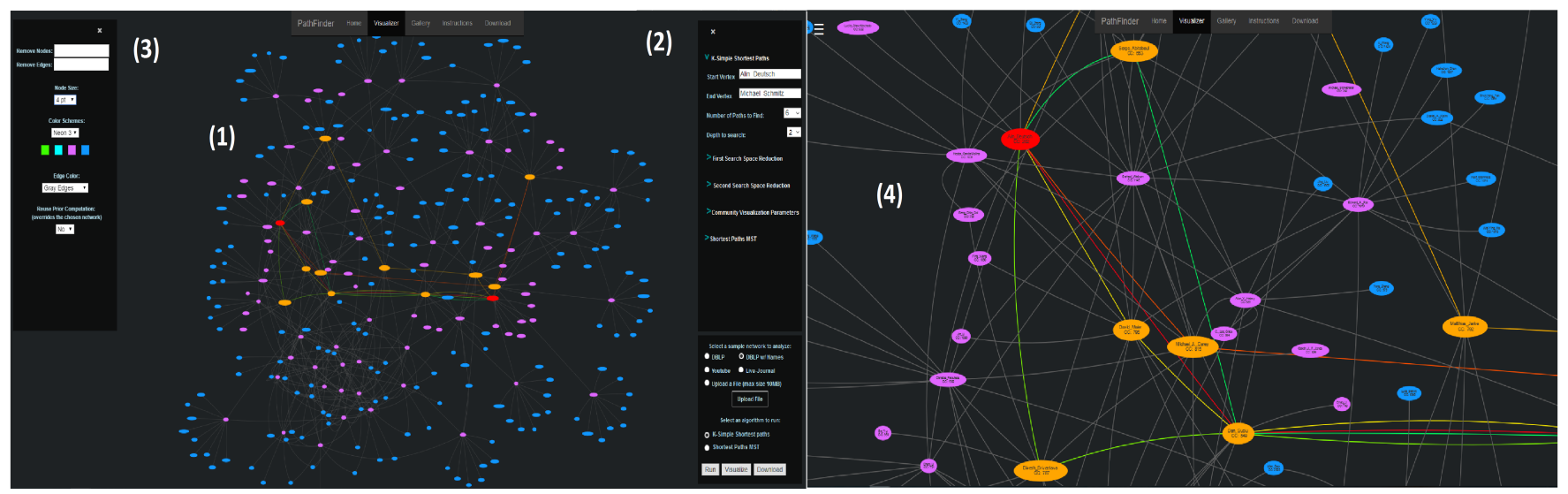

Figure 1: An illustrative example of our platform to find the key pathways. Start and end vertices are in red.

(4) Optional: Re-query the generated network with different vertices or find paths using the pathway detection algorithm

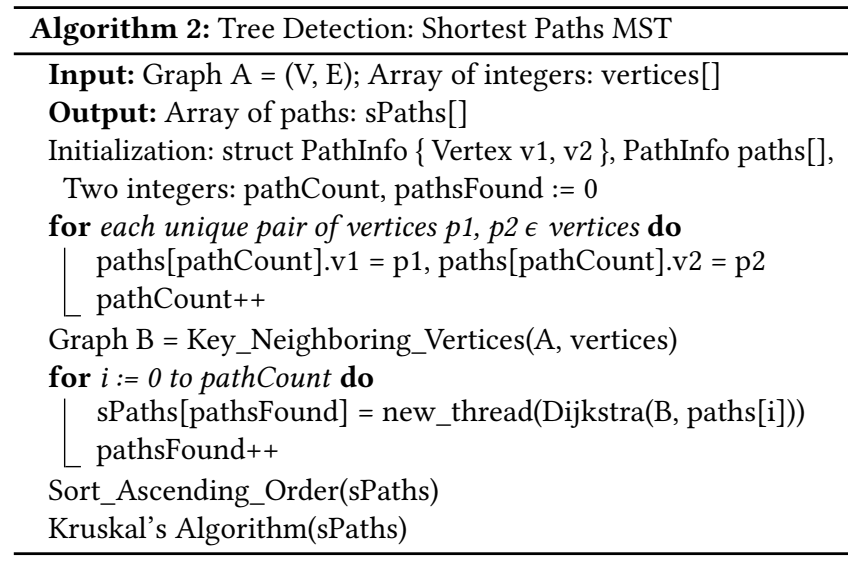

\subsection{Context \& Speed-up}

For both tree and pathway detection, we propose an efficient algorithm to detect key neighboring vertices to reduce the search space using a combination of three techniques to identify critical nodes: (1) vertex centrality, (2) edge connection to a pre-marked node and (3) breadth first search. This allows us to create a reduced graph $R=(V, E)$, that is a subset of $G, R \subset G$. Through this process we implicitly assume that vertices with high centrality are key hubs in the graph and are therefore important 'players' in the network.

The proposed key neighboring vertices algorithm can be viewed in 4 steps:

(1) Determine if the current vertex has an edge connection to one of the 'key' vertices and has above average vertex centrality. If both conditions are met, place the current vertex into a bin of that key vertex.

(2) Sort each bin in descending order of vertex centrality.

(3) From each bin, take the top ' $x$ ' neighboring nodes as important vertices at that depth level and add them to the reduced adjacency list.

(4) From each bin, a percentage of the top ' $x$ ' nodes will become 'key' vertices and recursively undergo the process until the specified depth level is reached.

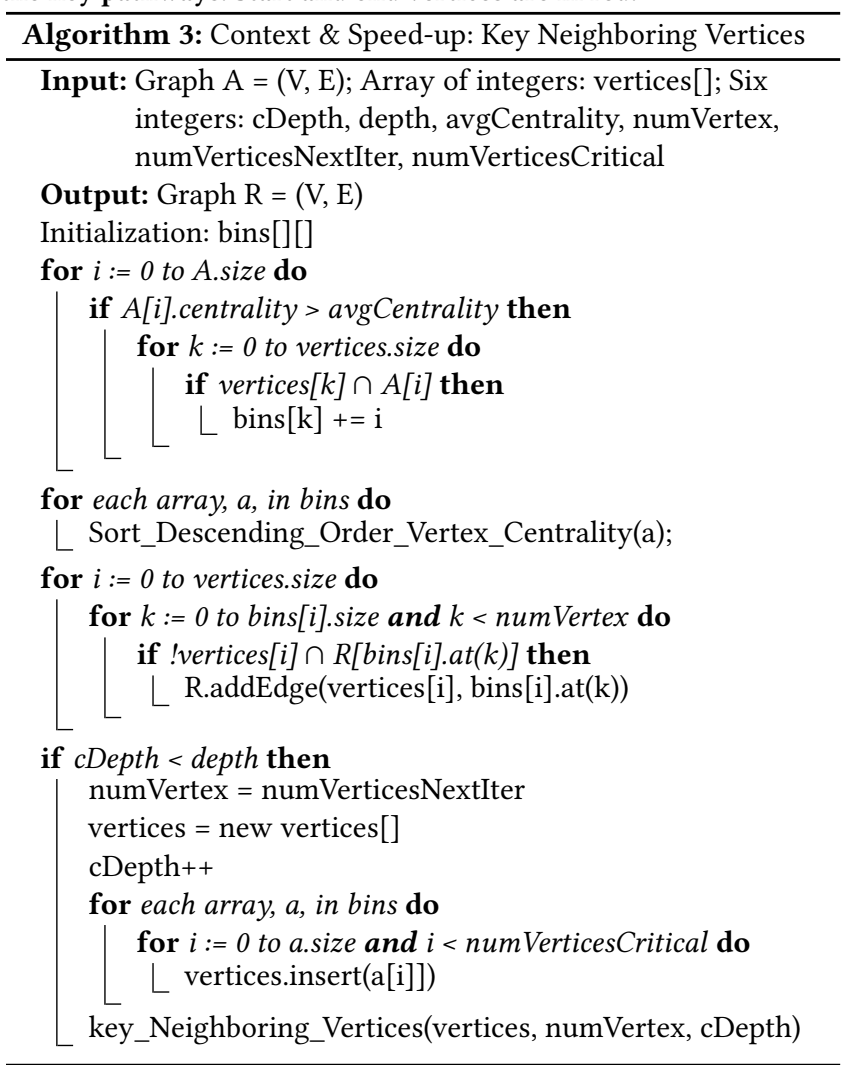

\subsection{Empirical Evaluation}

We used the DBLP co-authorship and the LiveJournal social network from the Stanford SNAP network to gather empirical data on the platform. The two measures we aim to quantify are speed and accuracy. Since the Shortest Paths MST and K-Simple Shortest Paths algorithm utilize the same search space reduction algorithm and multithreading techniques, we use the KSSP algorithm to represent the Shortest Paths MST in terms of accuracy and run time. All data was collected locally and does not account for any additional run time caused by using the web-platform. 
To compare the accuracy and run time, we ran three variations of the K-Simple Shortest Path algorithm (KSSP). The first variation (v.1) contained only the core KSSP algorithm with no search space reduction or multithreading. The second variation (v.2) ran the KSSP algorithm with multithreading (KSSPT). The third variation (v.3) ran the KSSP algorithm with multithreading and the search space reduction algorithm (KSSPR). The run time and accuracy of the three KSSP variations can be seen in Figure 2 and Figure 3-4 respectively. It should be noted that (v.1) and (v.2) of the KSSP algorithm will always find the shortest paths available, while the same guarantee cannot be extended to (v.3). The reasoning behind the possible suboptimal path(s) for (v.3) is due to the nature of the search space reduction algorithm applied to the graph. The KNV algorithm uses a tradeoff between accuracy and run time, which can be varied depending on the parameters. In trials for Figure 2 we applied parameters to the KNV algorithm that retained accuracy at the cost of speed. However, even with this additional 'cost', trial one results show (v.3) over $2.5 \mathbf{x}$ faster than (v.1) and $\mathbf{1 . 7 x}$ faster than (v.2) with no loss of accuracy with respect to the full network (last data point). It can be seen in Figure 4 that the run time and path length for the variations is dependent upon the start and end vertices.

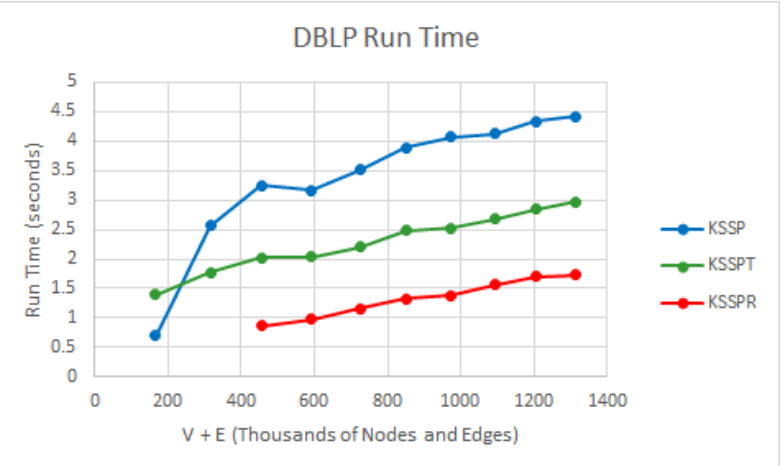

Figure 2: Start Vertex: 61, End Vertex: 70591, \# of paths: 6. No data for KSSPR on first two data points due to selected KNV parameters.

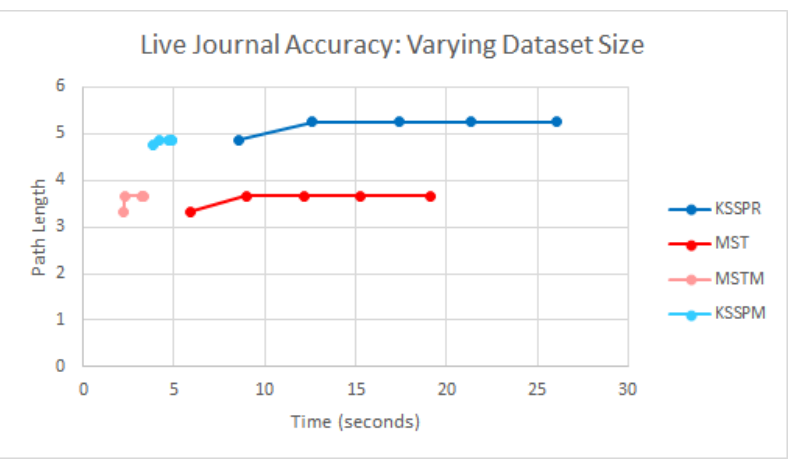

Figure 3: Data points represent $20 \%-100 \%$ of the Live-Journal network in 1/5th intervals. KSSPR: Start Vertex: 35521, End Vertex: 286345, \# of paths: 8. MST Shortest Paths: Vertices: 0, 58, 9558, 34343.

In order to better access the abilities of the platform we ran both the KSSPR and Shortest Paths MST algorithms on the LiveJournal network. To put it in perspective, the LiveJournal network has approximately 38.5 million edges and vertices compared to the DBLP

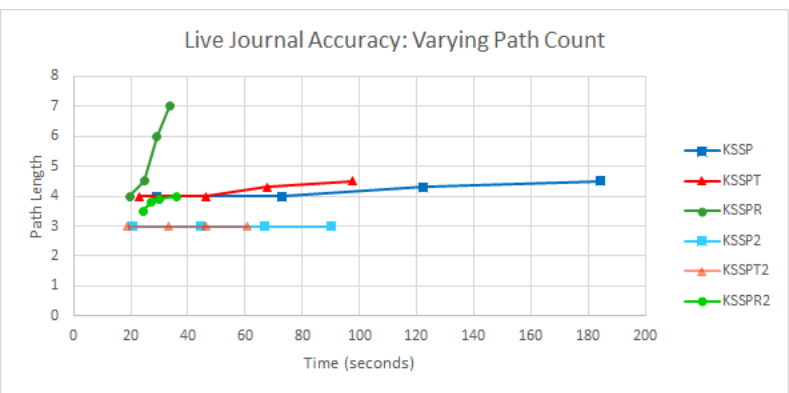

Figure 4: Each data point represents \# of paths found: 2-14 in intervals of 4. Trial 1: Start Vertex: 35521, End Vertex: 286345. Trial 2: Start Vertex: 9790, End Vertex: 26073.

network of 1.3 million. In figure 3, the labels KSSPM and MSTM represent re-query results compared to Algorithm 1 and 2 with no re-query. Comparing the full network (5th interval) run time for KSSPR vs KSSPM we see an 5.3x speed-up and a 5.6x speed-up for the MSTM vs MST. It should be noted that (a) parameters were held constant when re-querying the network and that only information generated from the previous run is analyzed when re-querying and (b) shorter paths can be found when re-querying the graph since it's saved based on visualization parameters not search space reduction.

\section{CONCLUSIONS}

The goal of this work is to rapidly analyze network connectivity. We believe the computational speedup obtained will be of interest to information management and data mining researchers. In addition, the web platform PathFinder allows users to quickly and intuitively determine network connectivity between a set of user-specific query nodes. An operational prototype is online: http: //path-finder.io and source code will be made publicly available by the conference date.

\section{ACKNOWLEDGEMENTS}

This work is partially supported by the National Science Foundation under Grant No. IIS-1651203, IIS-1715385 and IIS-1743040, by DTRA under the grant number HDTRA1-16-0017, by Army Research Office under the contract number W911NF-16-1-0168, and gifts from Huawei and Baidu.

\section{REFERENCES}

[1] L. Akoglu, D. H. Chau, J. Vreeken, N. Tatti, H. Tong, and C. Faloutsos. Mining Connection Pathways for Marked Nodes in Large Graphs. SDM 2013.

[2] C. L. Staudt, Y. Marrakchi, and H. Meyerhenke. Detecting Communities around Seed Nodes in Complex Networks. IEEE Big Data 2014.

[3] C. Faloutsos, K. S. Mccurley, and A. Tomkins. Fast Discovery of Connection Subgraphs. KDD 2004.

[4] E. Ruppert. 2000. Finding the k Shortest Paths in Parallel. Algorithmica 28, 2 (2000), 242-254.

[5] F. Guerriero, R. Musmanno. 2000. Parallel Asynchronous Algorithms for the K Shortest Paths Problem. JOTA 2000.

[6] A. Singh, D. Singh. 2015. Implementation of K-shortest Path Algorithm in GPU Using CUDA. Procedia Computer Science 48, 5-13.

[7] D. Sulieman, M. Malek, H. Kadima, D. Laurent. Semantic social breadth-first search and depth-first search recommendation algorithms.

[8] P. Cenek, M. Hrcka. Minimum Spanning Tree on A Subset of Nodes. 